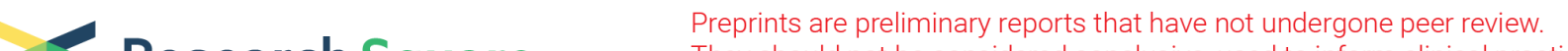 $\begin{array}{ll}\text { Research Square } & \begin{array}{l}\text { They should not be considered conclusive, used to inform clinical practice, } \\ \text { or referenced by the media as validated information. }\end{array}\end{array}$
}

\section{Low Serum Maresin-1 Levels Are Associated with Non-alcoholic Fatty Liver Disease: A Cross Sectional Study}

\section{Xia Fang}

The Affiliated Hospital of Southwest Medical University

Hongya Wang

The Affiliated Hospital of Southwest Medical University

Ting Ye

The Affiliated Hospital of Southwest Medical University

\section{Xiaolan Fu}

Yonchuan Hospital of Traditional Chinese Medicine

\section{Xiaozhen Tan}

The Affiliated Hospital of Southwest Medical University

\section{Yan Zeng}

The Affiliated Hospital of Southwest Medical University

\section{Jiahao Fan}

The Affiliated Hospital of Southwest Medical University

\section{Yong Xu ( $\nabla$ xywyll@aliyun.com )}

affiliated hospital of southwest medical university https://orcid.org/0000-0002-9534-6252

\section{Research Article}

Keywords: non-alcoholic fatty liver disease, Maresin-1, cross-sectional study

Posted Date: July 16th, 2021

DOI: https://doi.org/10.21203/rs.3.rs-714123/v1

License: (c) (1) This work is licensed under a Creative Commons Attribution 4.0 International License. Read Full License 


\section{Abstract \\ Background}

Maresin-1 is one of anti-inflammatory pro-resolving mediators, which is considered as a potential regulator of metabolic diseases. However, little information is available on the relationship between Maresin-1 and non-alcoholic fatty liver disease (NAFLD) in humans. Therefore, this study explored the associations between serum Maresin-1 levels and NAFLD.

\section{Methods}

A cross-sectional study was conducted in 240 Chinese people, including 116 non-NAFLD subjects and 124 NAFLD patients. NAFLD was diagnosed by abdominal ultrasonography. Serum Maresin-1 levels were determined by ELISA. The association between Maresin-1 and NAFLD was assessed.

\section{Results}

Serum Maresin-1 levels in NAFLD patients were markedly lower than those in non-NAFLD subjects (63.63 [59.87-73.93] vs 73.11 [65.12-84.50] pg/mL, $P=0.000$ ). The percentages of patients with NAFLD gradually decreased in tandem with increasing quartiles of Maresin-1 $(P<0.001)$. Furthermore, serum Maresin-1 levels were positively associated with AST/ALT, albumin, Albumin-globulin-ratio, and HDL-C (all $P<0.05)$ and negatively associated with $\mathrm{BMI}$, waist circumference, hip circumference, waist-to-hip ratio, ALT, GGT, uric acid, TG, and FBG (all $P<0.05$ ) after adjusting for sex and age. We found that serum Maresin-1 levels were significantly associated with NAFLD by binary logistic regression analysis.

\section{Conclusions}

Circulating Maresin-1 levels were decreased in patients with NAFLD, and there was a negative correlation between NAFLD and serum Maresin-1 concentrations. Decreased Maresin-1 might be involved in the development of NAFLD.

\section{Background}

Non-alcoholic fatty liver disease (NAFLD), which is the most common metabolic liver disease and one of the most common causes of chronic liver disease, is characterized by the ectopic deposition of fat in hepatocytes without secondary causes of liver fat accumulation (e.g., excess alcoholic consumption, medication, viral infection) [1]. NAFLD has a growing impact on world health. The global prevalence of NAFLD is estimated to be as high as $25 \%$, with the highest in the Middle East and South America and lowest in Africa [2]. Given the tremendous changes in lifestyle in the past 20 years, the prevalence of NAFLD in China has reached as high as $22.4 \%$, which is equivalent to that in the United State $(24.13 \%)$, 
Europe (23.71\%) and Japan (25\%) [2-6]. This rising prevalence of NAFLD will inflict a growing economic burden and will be accompanied by an increasing number of patients with cirrhosis, liver transplantation or/and Hepatocellular Carcinoma (HCC) [7-10]. However, NAFLD is a heterogeneous disease with very different clinical manifestations and different rates of progression among individuals. Some patients are asymptomatic and only found to have simple non-alcoholic fatty liver (NAFL) during physical examination, while others present with severe nonalcoholic steatohepatitis (NASH), liver cirrhosis, or even HCC. Therefore, it is necessary to identify any novel biomarkers in order to better investigate the progression of NAFLD and to screen out the high-risk groups who may be particularly susceptible to NAFLD.

Maresins are the third-largest family of specialized pro-resolving mediators (SPMs) made from docosahexaenoic acid (DHA), which are mainly biosynthesized in M2 macrophages [11-12]. Maresin-1 is the first discovered member of this family. Nowadays, more and more evidence show that Maresin-1 plays vital roles in metabolic diseases. In ob/ob and diet-induced obese mice, Maresin-1 improves insulin sensitivity and reverse adipose tissue dysfunction and inflammation [13]. Maresin-1 ameliorates liver steatosis by inhibiting endoplasmic reticulum stress and lipogenic enzymes and inducing autophagy via AMPK pathway in high fat diet (HFD)-fed mice [14-15]. A recent study by Han et al. showed that Maresin1 is an endogenous ligand of retinoic acid-related orphan receptor a (RORa). Maresin-1 protected mice from HFD-induced NASH by activating M2 polarization of liver macrophages in a RORa-dependent manner [16]. All these studies indicate that Maresin-1 is closely related to metabolic diseases, especially NAFLD in cells and animal models. However, the relationship between serum Maresin-1 levels and NAFLD in humans is still unclear.

In the present cross-sectional study, we recruited subjects with NAFLD to investigate alterations in circulating Maresin-1 and to explore the potential relationship between Maresin-1 levels and NAFLD subjects.

\section{Methods}

\section{Study Population and Design}

non-NAFLD subjects and NAFLD patients were recruited from the Physical Examination Center of the Affiliated Hospital of Southwest Medical University. All ultrasonographic examinations, using a Doppler sonography system (ACUSON Sequoia, SIEMENS, Germany), were performed to diagnose NAFLD by the same group of experienced sonographers following standardized procedures. The diagnosis of NAFLD was made based on the presence of hepatic fat accumulation according to the criteria issued by the Chinese Liver Disease Association [17]. All participants with the following conditions were excluded: 1) excess alcohol consumption ( $\geq 140 \mathrm{~g} /$ week for men, $\geq 70 \mathrm{~g} /$ week for women) [18] or alcoholic liver disease, drug- or toxin-induced liver diseases, genetic liver diseases, viral or autoimmune hepatitis, biliary obstructive diseases, renal disease, HIV infection, cancer, acute or chronic inflammatory disease, cardiovascular or cerebral vascular disease, or pregnancy or breastfeeding; 2) systemic corticosteroids 
treatment, anti-inflammatory therapy, hypoglycemic or lipid-lowering therapy, and antihypertensive treatment.

After screening, 124 patients with NAFLD and 116 subjects with non-NAFLD aged between 22 and 71 years participated in this clinical study. All participants were categorized into quartiles based on their serum Maresin-1 concentration: quartile 1, Maresin- $1<62.13 \mathrm{pg} / \mathrm{mL}$; quartile 2, $62.13 \mathrm{pg} / \mathrm{mL} \leq$ Maresin-1 $<68.71 \mathrm{pg} / \mathrm{mL}$; quartile 3, $68.71 \mathrm{pg} / \mathrm{mL} \leq$ Maresin-1 $\leq 77.79 \mathrm{pg} / \mathrm{mL}$; quartile 4, Maresin- $1>77.79 \mathrm{pg} / \mathrm{mL}$.

All experimental protocols followed the ethical guidelines of the 1964 Declaration of Helsinki and were approved by the Human Research Ethics Committee of the Affiliated Hospital of Southwest Medical University (permission no. KY2021086). Written informed consent was obtained from all participants.

\section{Anthropometric And Biochemical Measurements}

In the present study, all anthropometric measurements were performed before breakfast, and all participants wore light clothing and no footwear. After overnight fasting (about 10-12 h), the anthropometric parameters were measured by a designated specialist nurse. After resting for at least 5 minutes, systolic blood pressure (SBP) and diastolic blood pressure (DBP) were measured by using a medical automatic electronic sphygmomanometer (HBP-9020, OMRON Corp., Kyoto, Japan). Height and body weight (BW) were measured by using an ultrasonic analyzer (SK-V7, Shenzhen, China). Waist circumferences (WC, the midpoint between the ilium and the lowest margin of the ribs) and hip circumference ( $\mathrm{HC}$, the maximum circumference of the hips) were measured with a cloth measuring tape. Body mass index (BMI) and waist to hip ratio (WHR) were calculated as BW $(\mathrm{kg}) /$ height $\left(\mathrm{m}^{2}\right)$ and WC $(\mathrm{cm}) / \mathrm{HC}(\mathrm{cm})$, respectively.

After overnight fasting, blood samples were collected from all participants in the morning of the day of ultrasound examination. Serum samples were collected and stored at $-80^{\circ} \mathrm{C}$ until analysis. Fasting blood glucose (FBG), alanine aminotransferase (ALT), aspartate aminotransferase (AST), total protein (TP), albumin (ALB), globulin (GLO), gamma-glutamyl transpeptidase (GGT), alkaline phosphatase (ALP), urea nitrogen (Urea), uric acid (UA), creatinine (Crea), total cholesterol (TC), triglycerides (TG), high-density lipoprotein cholesterol (HDL-C), low-density lipoprotein cholesterol (LDL-C) and homocysteine (HCY) were detected by biochemical autoanalyzer (ADVIA2400, SIEMENS, Germany). Peripheral white blood cell (WBC) and neutrophils (NEU) counts were determined using an automated blood cell counter (Mindray BC-6800, Shenzhen, China).

\section{Serum Maresin-1 Measurement}

Serum Maresin-1 levels were quantified using commercial enzyme-linked immunosorbent assay (ELISA) kits (Human ELISA kit, Senbeijia, NanJing, China) according to the manufacture's protocol. Serum 
samples were diluted 5 -fold before the assay. The intra- and inter-assay variations were $<9 \%$ and $<11 \%$, respectively. The detectable range of the kit was $5 \mathrm{pg} / \mathrm{mL}-160 \mathrm{pg} / \mathrm{mL}$.

\section{Statistical Analysis}

SPSS 22.0 and GraphPad Prism 8.0 were used for all statistical analysis and graphics. Data were expressed as the number (percentage) for categorical variables or as the mean \pm standard deviation (SD) or medians [25th, 75th percentiles] for continuous variables, unless otherwise specified. The differences between the two groups were examined using the Student's t-test or Mann-Whitney U-test for continuous variables. The differences among more than two groups were performed with one-way analysis of variance (ANOVA) or Kruskal-Wallis test for continuous variables. Comparisons of categorical variables were made using Chi-square test. The correlation between serum Maresin-1 and other clinical parameters was determined by Pearson correlation or Spearman correlation test according to the distribution of parameters. Partial correlation coefficients were used for age- and sex-adjusted data. Binary logistic regression analyses were used to analyze the association between the serum Maresin-1 levels and NAFLD. $P$ value $<0.05$ was considered statistically significant.

\section{Results}

\section{General Characteristics and Serum Maresin-1 Levels of Study Participants}

A total of 116 non-NAFLD subjects and 124 NAFLD patients were enrolled in this study. The average age of the subjects was $43.44 \pm 11.36$ years, including 134 males (55.8\%) and 106 females (44.2\%). Table 1 summarizes the general anthropometric, biochemical and clinical parameters and serum Maresin- 1 levels of the subjects. Compared to non-NAFLD subjects, NAFLD patients had higher BMI, WC, HC, WHR, SBP, DBP, FBG, WBC count, ALT, AST, GGT, ALP, Urea, UA, Crea, TC, TG, LDL-C, and HCY $(P<0.05$ or $P<0.01$ or $P$ $<0.001$ ) and lower levels of AST/ALT and HDL-C (both $P<0.001$ ). The proportion of male was higher in subjects with NAFLD than in those non-NAFLD $(P=0.029)$. No significant differences were found in age, neutrophil count, TP, ALB, GLO, and A/G. 
Table 1

Main clinical parameters and serum Maresin-1 levels in all participants.

\begin{tabular}{|c|c|c|c|}
\hline Variables & non-NAFLD & NAFLD & $P$-value \\
\hline Male/Female & $57 / 59$ & $77 / 47$ & 0.029 \\
\hline Age (year) & $41.50(33.00-51.00)$ & $45.50(35.00-53.00)$ & 0.286 \\
\hline BMI (kg/m2) & $22.54(21.20-24.20)$ & $27.26(25.48-29.49)$ & 0.000 \\
\hline WC (cm) & $79.68 \pm 8.52$ & $90.39 \pm 7.92$ & 0.000 \\
\hline $\mathrm{HC}(\mathrm{cm})$ & $95.00(91.00-98.00)$ & $100.00(96.00-104.75)$ & 0.000 \\
\hline WHR & $0.84 \pm 0.06$ & $0.89 \pm 0.05$ & 0.000 \\
\hline $\mathrm{SBP}(\mathrm{mmHg})$ & $118.00(110.00-124.00)$ & $130.50(119.00-138.00)$ & 0.000 \\
\hline $\mathrm{DBP}(\mathrm{mmHg})$ & $71.45 \pm 9.21$ & $77.29 \pm 10.68$ & 0.000 \\
\hline FBG (mmol/L) & $4.95(4.68-5.23)$ & $5.32(4.90-5.75)$ & 0.000 \\
\hline WBC $\left(* 10^{\wedge} 9 / \mathrm{L}\right)$ & $6.11 \pm 1.22$ & $6.75 \pm 1.35$ & 0.000 \\
\hline $\operatorname{NEU}\left(* 10^{\wedge} 9 / \mathrm{L}\right)$ & $3.65 \pm 0.92$ & $3.85 \pm 0.95$ & 0.099 \\
\hline $\mathrm{ALT}(\mathrm{U} / \mathrm{L})$ & $18.20(13.30-27.20)$ & $27.90(21.25-42.05)$ & 0.000 \\
\hline AST (U/L) & $20.20(17.30-26.05)$ & $23.40(19.43-27.50)$ & 0.002 \\
\hline AST/ALT & $1.15(0.92-1.34)$ & $0.83(0.66-0.99)$ & 0.000 \\
\hline $\mathrm{TP}(\mathrm{g} / \mathrm{L})$ & $73.00 \pm 3.01$ & $72.63 \pm 2.93$ & 0.328 \\
\hline $\operatorname{ALB}(g / L)$ & $46.25(45.00-47.63)$ & $46.05(45.03-47.80)$ & 0.970 \\
\hline GLO (g/L) & $26.62 \pm 2.52$ & $26.25 \pm 2.59$ & 0.260 \\
\hline$A / G$ & $1.76(1.62-1.90)$ & $1.77(1.65-1.91)$ & 0.501 \\
\hline GGT (U/L) & 16.05 (13.10-26.85) & $31.55(20.58-48.60)$ & 0.000 \\
\hline ALP (U/L) & $67.40(53.95-79.78)$ & $74.05(62.55-88.05)$ & 0.003 \\
\hline Urea (mol/L) & $4.70(3.90-5.63)$ & $5.13(4.59-5.93)$ & 0.004 \\
\hline $\mathrm{UA}(\mu \mathrm{mol} / \mathrm{L})$ & $320.00(261.48-380.78)$ & $370.15(316.65-440.00)$ & 0.000 \\
\hline
\end{tabular}

Continuous variables are mean \pm standard deviation (SD) or medians (25th, 75th percentiles). NAFLD: non-alcoholic fatty liver disease, BMI: body mass index, WC: waist circumference, HC: hip circumference, WHR: waist to hip ratio, SBP: systolic blood pressure, DBP: diastolic blood pressure, FBG: fasting blood glucose, WBC: white blood cells, NEU: neutrophil, ALT: alanine aminotransferase, AST: aspartate aminotransferase, TP: total protein, ALB: albumin, GLO: globulin, A/G: Albuminglobulin-ratio, GGT: gamma-glutamyl transpeptidase, ALP: alkaline phosphatase, UA: uric acid, Crea: creatinine, TC: total cholesterol, TG: triglyceride, HDL-C: high-density lipoprotein cholesterol, LDL-C: low-density lipoprotein cholesterol, HCY: Homocysteine. 


\begin{tabular}{|c|c|c|c|}
\hline Variables & non-NAFLD & NAFLD & $P$-value \\
\hline Crea $(\mu \mathrm{mol} / \mathrm{L})$ & $63.35(53.80-71.40)$ & $70.50(55.75-77.23)$ & 0.006 \\
\hline $\mathrm{TC}(\mathrm{mmol} / \mathrm{L})$ & $4.73 \pm 0.77$ & $4.95 \pm 0.72$ & 0.022 \\
\hline TG (mmol/L) & $1.16(0.84-1.67)$ & $2.00(1.42-2.67)$ & 0.000 \\
\hline HDL-C (mmol/L) & $1.35(1.17-1.66)$ & $1.08(0.96-1.22)$ & 0.000 \\
\hline LDL-C (mmol/L) & $3.10 \pm 0.83$ & $3.42 \pm 0.70$ & 0.002 \\
\hline $\mathrm{HCY}(\mu \mathrm{mol} / \mathrm{L})$ & $9.55(8.00-11.50)$ & $10.65(8.80-12.10)$ & 0.009 \\
\hline Maresin-1 $(\mathrm{pg} / \mathrm{mL})$ & $73.11(65.12-84.50)$ & $63.63(59.87-73.93)$ & 0.000 \\
\hline \multicolumn{4}{|c|}{$\begin{array}{l}\text { Continuous variables are mean } \pm \text { standard deviation (SD) or medians (25th, } 75 \text { th percentiles). NAFLD } \\
\text { non-alcoholic fatty liver disease, BMI: body mass index, WC: waist circumference, HC: hip } \\
\text { circumference, WHR: waist to hip ratio, SBP: systolic blood pressure, DBP: diastolic blood pressure, } \\
\text { FBG: fasting blood glucose, WBC: white blood cells, NEU: neutrophil, ALT: alanine aminotransferase, } \\
\text { AST: aspartate aminotransferase, TP: total protein, ALB: albumin, GLO: globulin, A/G: Albumin- } \\
\text { globulin-ratio, GGT: gamma-glutamyl transpeptidase, ALP: alkaline phosphatase, UA: uric acid, Crea: } \\
\text { creatinine, TC: total cholesterol, TG: triglyceride, HDL-C: high-density lipoprotein cholesterol, LDL-C: } \\
\text { low-density lipoprotein cholesterol, HCY: Homocysteine. }\end{array}$} \\
\hline
\end{tabular}

In all study subjects, the distribution of serum Maresin-1 concentration was from $51.65-224.45 \mathrm{pg} / \mathrm{mL}$. There was no significant difference in circulating Maresin-1 levels between men and women (66.99 [61.18-77.40] vs 71.52 [62.57-79.32], $P=0.078$ ). In addition, in order to understand the serum levels of Maresin-1 under different metabolic conditions, all participants were divided into normal and abnormal groups according to the levels of FBG, lipids or BMI $[19,20]$. As shown in Fig. 1, serum Maresin-1 levels were significantly lower in subjects with elevated FBG, TC and TG levels (all $P<0.001$, Fig. 1A-C), decreased HDL-C levels ( $P<0.001$; Fig. 1D), and overweight or obese subjects $(P<0.001$; Fig. 1F) compared to their controls. There was no significant difference in serum Maresin-1 levels among subjects with different LDL-C levels ( $P=0.2587$; Fig. 1E). Importantly, serum Maresin-1 levels in NAFLD patients were significantly decreased compared with those in non-NAFLD subjects $(P=0.000$, Table 1$)$.

\section{Clinical and biochemical characteristics by quartiles of serum Maresin-1 in all study participants}

Table 2 shows the clinical and biochemical characteristics according to quartiles of serum Maresin-1 levels in all subjects. BMI, WC, HC, WHR, SBP, DBP, FBG, ALT, AST, AST/ALT, GLO, A/G, GGT, ALP, Urea, TG, HDL-C, and Maresin-1 concentrations were significantly different between participants in different serum Maresin-1 quartiles $(P<0.05$ or $P<0.01$ or $P<0.001)$. Compared to subjects in the lowest quartile of serum Maresin-1 concentration, participants in the highest quartile had lower levels of BMI, WC, HC, WHR, SBP, DBP, FBG, WBC count, ALT, GLO, GGT, Urea, TC, TG, and LDL-C $(P<0.05$ or $P<0.01$ or $P<0.001)$ and higher levels of serum Maresin-1, AST/ALT, A/G, and HDL-C $(P<0.01$ or $P<0.001)$. As shown in Fig. 2, the prevalence of NAFLD was rapidly decreased in tandem with increasing quartile of serum Maresin-1 levels $(P<0.001)$. 
Table 2

Clinical and biochemical characteristics by quartile of serum Maresin-1 level in all subjects.

\begin{tabular}{|c|c|c|c|c|c|}
\hline Variables & Quartile 1 & Quartile 2 & Quartile 3 & Quartile 4 & $\begin{array}{l}P \text { - } \\
\text { value }\end{array}$ \\
\hline & $(\sim<62.13)$ & $\begin{array}{l}(62.13 \leq-< \\
68.71)\end{array}$ & $\begin{array}{l}(68.71 \leq-< \\
77.79)\end{array}$ & $(\sim \geq 77.79)$ & \\
\hline Sample Size & 60 & 60 & 60 & 60 & - \\
\hline Male/Female & $37 / 23$ & $38 / 22$ & $28 / 32$ & $31 / 29$ & 0.198 \\
\hline Age (year) & $\begin{array}{l}46.00(40.25- \\
52.75)\end{array}$ & $\begin{array}{l}46.00(34.00- \\
54.00)\end{array}$ & $\begin{array}{l}38.50(31.00- \\
51.75)\end{array}$ & $\begin{array}{l}40.00(31.25- \\
49.75)\end{array}$ & 0.075 \\
\hline BMI (kg/m2) & $\begin{array}{l}26.13(24.19- \\
28.93)\end{array}$ & $\begin{array}{l}26.27(23.75- \\
28.25)\end{array}$ & $\begin{array}{l}25.23(21.99- \\
27.89)\end{array}$ & $\begin{array}{l}23.06(21.17- \\
25.60) * \star \star \star\end{array}$ & 0.000 \\
\hline WC (cm) & $89.20 \pm 9.73$ & $87.13 \pm 9.39$ & $83.67 \pm 9.29$ ** & 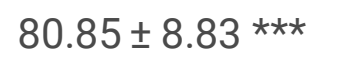 & 0.000 \\
\hline $\mathrm{HC}(\mathrm{cm})$ & $\begin{array}{l}99.00(94.25- \\
104.00)\end{array}$ & $\begin{array}{l}98.00(93.00- \\
102.00)\end{array}$ & $\begin{array}{l}97.50(93.25- \\
103.00)\end{array}$ & $\begin{array}{l}95.00(92.00- \\
99.00) *\end{array}$ & 0.017 \\
\hline WHR & $\begin{array}{l}0.90(0.88- \\
0.93)\end{array}$ & $\begin{array}{l}0.89(0.84- \\
0.92)\end{array}$ & $\begin{array}{l}0.85(0.81- \\
0.90) \text { ** }\end{array}$ & $0.85(0.80-0.88)$ & 0.000 \\
\hline $\mathrm{SBP}(\mathrm{mmHg})$ & $\begin{array}{l}130.50(118.00- \\
137.00)\end{array}$ & $\begin{array}{l}123.00(115.00- \\
134.75)\end{array}$ & $\begin{array}{l}121.50(111.00- \\
134.75)\end{array}$ & $\begin{array}{l}120.00(112.00- \\
129.50) *\end{array}$ & 0.038 \\
\hline $\mathrm{DBP}(\mathrm{mmHg})$ & $76.93 \pm 10.84$ & $75.95 \pm 10.51$ & $73.78 \pm 10.31$ & $71.20 \pm 9.15$ ** & 0.012 \\
\hline $\begin{array}{l}\text { FBG } \\
(\mathrm{mmol} / \mathrm{L})\end{array}$ & $\begin{array}{l}5.47(4.86- \\
5.81)\end{array}$ & $\begin{array}{l}5.09(4.83- \\
5.68)\end{array}$ & $\begin{array}{l}5.06(4.73- \\
5.38) \star\end{array}$ & $\underset{\star \star}{4.98}(4.68-5.27)$ & 0.001 \\
\hline $\begin{array}{l}\text { WBC } \\
\left(* 10^{\wedge} 9 / \mathrm{L}\right)\end{array}$ & $6.72 \pm 1.45$ & $6.59 \pm 1.33$ & $6.30 \pm 1.17$ & $6.17 \pm 1.29$ * & 0.079 \\
\hline $\begin{array}{l}\text { NEU } \\
\left(* 10^{\wedge} 9 / L\right)\end{array}$ & $\begin{array}{l}3.67(3.08- \\
4.47)\end{array}$ & $\begin{array}{l}3.69(3.07- \\
4.58)\end{array}$ & $\begin{array}{l}3.55(3.11- \\
4.12)\end{array}$ & $3.67(2.99-4.43)$ & 0.830 \\
\hline $\operatorname{ALT}(\mathrm{U} / \mathrm{L})$ & $\begin{array}{l}26.70(19.45- \\
38.18)\end{array}$ & $\begin{array}{l}27.40(19.48- \\
36.70)\end{array}$ & $\begin{array}{l}20.90(13.33- \\
30.35) *\end{array}$ & $\begin{array}{l}19.20(13.98- \\
27.88) * *\end{array}$ & 0.000 \\
\hline AST (U/L) & $\begin{array}{l}23.20(19.45- \\
29.63)\end{array}$ & $\begin{array}{l}23.30(19.98- \\
28.40)\end{array}$ & $\begin{array}{l}20.15(16.83- \\
26.38)\end{array}$ & $\begin{array}{l}20.15(17.53- \\
24.78)\end{array}$ & 0.010 \\
\hline AST/ALT & $\begin{array}{l}0.85(0.67- \\
1.06)\end{array}$ & $\begin{array}{l}0.88(0.67- \\
1.14)\end{array}$ & $\begin{array}{l}1.02(0.83- \\
1.21) *\end{array}$ & ${ }_{\star \star}^{1.11(0.85-1.28)}$ & 0.000 \\
\hline $\mathrm{TP}(\mathrm{g} / \mathrm{L})$ & $73.10 \pm 2.88$ & $73.01 \pm 2.54$ & $72.20 \pm 3.37$ & $72.95 \pm 3.00$ & 0.321 \\
\hline ALB $(g / L)$ & $\begin{array}{l}45.95(45.00- \\
47.00)\end{array}$ & $\begin{array}{l}45.85(45.10- \\
47.78)\end{array}$ & $\begin{array}{l}45.90(44.90- \\
47.48)\end{array}$ & $\begin{array}{l}47.25(45.33- \\
48.83)\end{array}$ & 0.058 \\
\hline
\end{tabular}

Continuous variables are mean \pm standard deviation or medians (25th, 75 th percentiles). ${ }^{\star} P<0.05$, $\star \star * P<0.01,{ }^{* \star *} P<0.001$ versus quartile 1 group. 


\begin{tabular}{|c|c|c|c|c|c|}
\hline Variables & Quartile 1 & Quartile 2 & Quartile 3 & Quartile 4 & $\begin{array}{l}P \\
\text { value }\end{array}$ \\
\hline GLO (g/L) & $27.11 \pm 3.05$ & $26.64 \pm 2.34$ & $26.01 \pm 2.44$ * & $25.97 \pm 2.20$ * & 0.039 \\
\hline$A / G$ & $\begin{array}{l}1.72(1.55- \\
1.83)\end{array}$ & $\begin{array}{l}1.73(1.60- \\
1.88)\end{array}$ & $\begin{array}{l}1.79(1.66- \\
1.91)\end{array}$ & ${ }_{\star}^{1.81}(1.73-1.94)$ & 0.009 \\
\hline GGT (U/L) & $\begin{array}{l}30.90(19.23- \\
47.33)\end{array}$ & $\begin{array}{l}29.10(18.03- \\
42.53)\end{array}$ & $\begin{array}{l}20.35(14.68- \\
31.40) *\end{array}$ & $\begin{array}{l}15.40(12.68- \\
28.30) \star \star \star\end{array}$ & 0.000 \\
\hline ALP (U/L) & $\begin{array}{l}73.85(56.45- \\
84.73)\end{array}$ & $\begin{array}{l}73.80(62.43- \\
84.70)\end{array}$ & $\begin{array}{l}72.15(61.23- \\
87.53)\end{array}$ & $\begin{array}{l}63.20(52.40- \\
79.13)\end{array}$ & 0.031 \\
\hline Urea (mol/L) & $\begin{array}{l}5.16(4.83- \\
5.96)\end{array}$ & $\begin{array}{l}4.98(4.40- \\
5.90)\end{array}$ & $\begin{array}{l}4.84(3.97- \\
5.77)\end{array}$ & $\underset{*}{4.76}(3.96-5.41)$ & 0.027 \\
\hline $\mathrm{UA}(\mu \mathrm{mol} / \mathrm{L})$ & $\begin{array}{l}356.55(296.10- \\
418.60)\end{array}$ & $\begin{array}{l}362.35(314.58- \\
434.23)\end{array}$ & $\begin{array}{l}332.80(283.48- \\
400.93)\end{array}$ & $\begin{array}{l}318.55(269.15- \\
415.45)\end{array}$ & 0.160 \\
\hline $\begin{array}{l}\text { Crea } \\
(\mu \mathrm{mol} / \mathrm{L})\end{array}$ & $\begin{array}{l}68.70(55.95- \\
75.25)\end{array}$ & $\begin{array}{l}66.70(54.38- \\
74.38)\end{array}$ & $\begin{array}{l}65.40(52.63- \\
73.78)\end{array}$ & $\begin{array}{l}65.20(54.93- \\
74.95)\end{array}$ & 0.735 \\
\hline $\mathrm{TC}(\mathrm{mmol} / \mathrm{L})$ & $5.00 \pm 0.80$ & $4.92 \pm 0.75$ & $4.74 \pm 0.77$ & $4.71 \pm 0.67$ * & 0.098 \\
\hline TG (mmol/L) & $\begin{array}{l}1.81(1.28- \\
2.65)\end{array}$ & $\begin{array}{l}1.79(1.23- \\
2.63)\end{array}$ & $\begin{array}{l}1.37(0.91- \\
1.82) \star \star\end{array}$ & $1.21(0.90-1.69)$ & 0.000 \\
\hline $\begin{array}{l}\mathrm{HDL}-\mathrm{C} \\
(\mathrm{mmol} / \mathrm{L})\end{array}$ & $\begin{array}{l}1.10(0.99- \\
1.34)\end{array}$ & $\begin{array}{l}1.11(0.96- \\
1.24)\end{array}$ & $\begin{array}{l}1.24(1.08- \\
1.46)\end{array}$ & ${ }_{\star \star \star}^{1.33}(1.17-1.63)$ & 0.000 \\
\hline $\begin{array}{l}\text { LDL-C } \\
(\mathrm{mmol} / \mathrm{L})\end{array}$ & $3.42 \pm 0.79$ & $3.32 \pm 0.82$ & $3.24 \pm 0.77$ & $3.07 \pm 0.72$ * & 0.093 \\
\hline $\begin{array}{l}\mathrm{HCY} \\
(\mu \mathrm{mol} / \mathrm{L})\end{array}$ & $\begin{array}{l}10.05(8.40- \\
11.78)\end{array}$ & $\begin{array}{l}10.10(8.60- \\
12.20)\end{array}$ & $\begin{array}{l}10.10(8.70- \\
11.90)\end{array}$ & $\begin{array}{l}10.15(7.63- \\
12.10)\end{array}$ & 0.866 \\
\hline
\end{tabular}

\section{Association of Serum Maresin-1 Levels with Clinical Parameters in the Study Population}

Next, correlation analysis was performed to investigate the association of serum Maresin-1 levels and other clinical parameters. As shown in Table 3, in all study population, serum Maresin-1 concentrations were positively associated with AST/ALT, ALB, A/G, and HDL-C $(P<0.05$ or $P<0.01$ or $P<0.001)$ and were negatively associated with Age, BMI, SBP, WC, HC, WHR, WBC count, ALT, AST, GLO, GGT, Urea, TG, LDL-C, and FBG $(P<0.05$ or $P<0.01$ or $P<0.001)$. After adjusting for sex and age, Maresin-1 remained statistically positively associated with AST/ALT, ALB, A/G, and HDL-C $(P<0.05$ or $P<0.01$ or $P<0.001)$ and negatively associated with BMI, WC, HC, WHR, ALT, GGT, UA, TG, and FBG $(P<0.05$ or $P<0.01$ or $P<$ $0.001)$. 
Table 3

The correlations analysis of variables associated with serum Maresin-1 levels in study population.

\begin{tabular}{|c|c|c|c|c|}
\hline & \multicolumn{2}{|c|}{ Serum Maresin-1 } & \multicolumn{2}{|c|}{ Serum Maresin-1 (age- and sex-adjusted) } \\
\hline & r & $P$-value & $r$ & $P$-value \\
\hline Sex & 0.114 & 0.078 & - & - \\
\hline Age & -0.171 & 0.008 & - & - \\
\hline BMI & -0.331 & 0.000 & -0.318 & 0.000 \\
\hline WC & -0.329 & 0.000 & -0.294 & 0.000 \\
\hline $\mathrm{HC}$ & -0.204 & 0.001 & -0.224 & 0.000 \\
\hline WHR & -0.360 & 0.000 & -0.281 & 0.000 \\
\hline SBP & -0.211 & 0.001 & -0.093 & 0.152 \\
\hline DBP & -0.113 & 0.081 & -0.079 & 0.222 \\
\hline FBG & -0.285 & 0.000 & -0.186 & 0.004 \\
\hline WBC & -0.155 & 0.016 & -0.093 & 0.151 \\
\hline NEU & -0.060 & 0.352 & -0.015 & 0.823 \\
\hline ALT & -0.289 & 0.000 & -0.212 & 0.001 \\
\hline AST & -0.214 & 0.001 & -0.102 & 0.116 \\
\hline AST/ALT & 0.260 & 0.000 & 0.240 & 0.000 \\
\hline TP & 0.016 & 0.810 & 0.006 & 0.931 \\
\hline ALB & 0.157 & 0.015 & 0.172 & 0.008 \\
\hline GLO & -0.150 & 0.020 & -0.123 & 0.058 \\
\hline$A / G$ & 0.204 & 0.002 & 0.156 & 0.016 \\
\hline GGT & -0.358 & 0.000 & -0.158 & 0.015 \\
\hline ALP & -0.119 & 0.065 & -0.085 & 0.193 \\
\hline Urea & -0.211 & 0.001 & -0.125 & 0.055 \\
\hline UA & -0.126 & 0.051 & -0.129 & 0.048 \\
\hline Crea & -0.068 & 0.294 & -0.038 & 0.559 \\
\hline TC & -0.089 & 0.169 & -0.072 & 0.270 \\
\hline TG & -0.330 & 0.000 & -0.192 & 0.003 \\
\hline
\end{tabular}




\begin{tabular}{|lcccc|}
\hline & \multicolumn{2}{c|}{ Serum Maresin-1 } & \multicolumn{2}{c|}{ Serum Maresin-1 (age- and sex-adjusted) } \\
\hline HDL-C & 0.255 & 0.000 & 0.242 & 0.000 \\
\hline LDL-C & -0.150 & 0.020 & -0.121 & 0.062 \\
HCY & 0.002 & 0.977 & -0.013 & 0.837 \\
\hline
\end{tabular}

Further, binary logistic regression analysis was performed to investigate the association of Maresin-1 with NAFLD. When no adjustment was made, serum Maresin-1 levels were significantly and inversely associated with the prevalence of NAFLD [OR $=0.945 ; 95 \% \mathrm{Cl}=0.922-0.968, P=0.000]$. After adjusting for sex, age, BMI, SBP, DBP, WHR, and FBG or further adjusting for ALT, AST, AST/ALT and UA, the association between serum Maresin-1 levels and the presence of NAFLD was not affected [OR $=0.958$, $95 \% \mathrm{Cl}=0.928-0.989, P=0.008]$ or $[\mathrm{OR}=0.957,95 \% \mathrm{Cl}=0.926-0.989, P=0.008]$. Finally, even after adjusting for lipid profiles (TC, TG, HDL-C, and LDL-C), the Maresin-1 levels were significantly associated with NAFLD [OR $=0.965,95 \% \mathrm{Cl}=0.933-0.999, P=0.042]$.

\section{Discussion}

Maresin-1 has been identified as a new member of pro-inflammatory regression mediators with an important role in metabolic disorders diseases, including NAFLD [14-16, 21]. However, there is limited evidence to support the potential role of Maresin-1 in the occurrence and development of NAFLD in humans. In the present cross-sectional study, we found that serum Maresin-1 levels were significantly decreased in NAFLD patients compared with those in non-NAFLD subjects. The prevalence of NAFLD was significantly decreased in participants with the highest serum Maresin-1 quartile than in participants with the lowest serum Maresin-1 quartile. In addition, our study demonstrated that serum Maresin-1 concentrations were positively correlated with parameters regarding AST/ALT, ALB, A/G, and HDL-C and negatively correlated with age, obesity, FBG, ALT, GGT, UA, and TG. Importantly, we found that serum Maresin-1 levels were independently associated with NAFLD after adjusting for other potential confounders. Overall, these data indicate that Maresin-1 is an independent protective factor of NAFLD, and the decrease of Maresin-1 levels may play a vital role in the pathophysiological process of NAFLD.

Maresin-1 was the first member of maresins family to be identified. The biosynthesis of maresins mainly occurs in M2 macrophages and is initiated by the key enzyme 12-lipoxygenase (12-LOX) [22-23]. DHA in macrophages is converted to 13S,14S-epoxy-maresin under the action of 12-LOX. Next, the epoxide intermediate forms the final product 7R, 14S-dihydroxydocosa-4Z, 8E, 10E, 12Z, 16Z, 19Z-hexaenoic acid (Maresin-1) through an epoxide-hydrolysis reaction [24]. In NAFLD, activated Kupffer cells release proinflammatory cytokines and chemokines [25], which promote the accumulation of pro-inflammatory M1polarized monocytes in liver tissue [26]. This disrupts the M1/M2 balance, resulting in a decrease in M2 pro-resolving phenotype in liver tissue. Han et al. found that Maresin-1, as an endogenous ligand of $\mathrm{RORa}$, increased the M2 polarity of liver macrophages by enhancing the expression and transcriptional activity of RORa [16]. Collectively, Maresin-1 is synthesized in M2 macrophages and promotes

Page $11 / 18$ 
macrophages to shift to M2 pro-resolving phenotype. When NAFLD occurs, the above loop is destroyed, resulting in the decrease of Maresin-1 synthesis. Our study found that serum Maresin-1 levels in NAFLD patients were significantly decreased than that in non-NAFLD subjects, and with the increase of serum level of Maresin-1, the prevalence of NAFLD decreased. Binary logistic regression analyses showed that Maresin-1 levels were an independent predictor of NAFLD. Thus, these results also suggest that Maresin-1 may be causal factor of NAFLD.

It is well known that NAFLD is a hepatic phenotype of metabolic impairment [27], and our current results are consistent with previous reports [28], which revealed that NAFLD patients had abnormal metabolic features. In the present study, the NAFLD patients had significantly higher BMI, WC, HC, WHR, BP, FBG, ALT, AST, GGT, ALP, Urea, UA, Crea, TC, LDL-C, TG, and HCY levels, but lower AST/ALT ratios and HDL-C than those with non-NAFLD subjects. In addition, our study results showed that serum Maresin-1 levels were negatively associated with BMI, WC, HC, WHR, FBG, ALT, GGT, UA, and TG and positively associated with AST/ALT, ALB, A/G, and HDL-C. Moreover, Maresin-1 has been reported to ameliorate insulin resistance (IR) and dyslipidemia by stimulating the insulin signaling [15, 29-30]. Overall, these results suggest that serum Maresin-1 level is negatively associated with metabolic disorder, indicating that the decrease of serum Maresin-1 may promote the occurrence of NAFLD by aggravating IR and lipid metabolic disorder.

Recently, Félix-Soriano et al. found that Maresin-1 was significantly reduced in adipose tissue in the aged HFD-induced obese mice when compared to the young normal controls [31]. Markworth et al. also showed that the aged mice muscle displayed significantly lower Maresin-1 levels when compared to the young mice muscle [32]. These data suggested that aging is associated with a deficiency of Maresin-1. Our results also showed that serum Maresin-1 levels were significantly negatively correlated with age in all participants.

A couple of limitations of our study should be mentioned. First, the current cross-sectional study is unable to illustrate the causal relationship between the serum Maresin-1 concentration and NAFLD, and it needs to be complemented by prospective studies. Second, we used abdominal ultrasound instead of liver biopsy to diagnosis NAFLD, which can lead to missed or misdiagnosis. Although hepatic steatosis, as an early stage of NAFLD, can be reliably identified by non-invasively imaging tests, including ultrasound, the advanced NAFLD such as NASH and cirrhosis can only be definitely diagnosed by liver biopsy [33]. Thus, the lack of histological confirmation of the degree of hepatic steatosis could be another weakness of the current study. Third, there might exist a selection bias. All the participants, who were recruited from physical examination center, as such, they may have stronger health awareness than non-participants. Finally, our current study may underestimate the true association, because serum Maresin-1 level was only detected once by ELISA kit, which is prone to random measurement error.

\section{Conclusions}


In conclusion, our study provides clinical evidence that the serum Maresin-1 concentrations were decreased in NAFLD patients, and that high serum Maresin-1 levels were associated with a reduced prevalence of NAFLD. These findings indicated that Maresin-1 might be a non-invasive molecular biomarker that detects the presence of NAFLD. However, future prospective longitudinal studies are required to confirm the contribution of Maresin-1 to the development of NAFLD.

\section{Abbreviations}

NAFLD, non-alcoholic fatty liver disease; NAFL, non-alcoholic fatty liver; NASH, non-alcoholic steatohepatitis; BMI, body mass index; WC, waist circumference; HC, hip circumference; WHR, waist to hip ratio; SBP, systolic blood pressure; DBP, diastolic blood pressure; FBG, fasting blood glucose; WBC, white blood cells; NEU, neutrophil; ALT, alanine aminotransferase; AST, aspartate aminotransferase; TP, total protein; ALB, albumin; GLO, globulin; A/G, Albumin-globulin-ratio; GGT, gamma-glutamyl transpeptidase; ALP, alkaline phosphatase; UA, uric acid; Crea, creatinine; TC, total cholesterol; TG, triglyceride; HDL-C, high-density lipoprotein cholesterol; LDL-C, low-density lipoprotein cholesterol; HCY, Homocysteine.

\section{Declarations}

\section{Ethics approval and consent to participate}

The Human Research Ethics Committee of the Affiliated Hospital of Southwest Medical University approved the experimental protocols (permission no. KY2021086). Written informed consent was obtained from all participants.

\section{Consent for publication}

Not applicable.

\section{Availability of data and materials}

The datasets used and/or analyzed during the current study are available from the corresponding author on reasonable request.

\section{Competing interests}

The authors declare that they have no competing interests.

\section{Funding}

This work was supported by the Doctor Initiation Fund of the Affiliated Hospital of Southwest Medical University [grant no. 19064], the Key Project of Natural Science of Southwest Medical University [grant no. 2020ZRZD004], and the Sichuan Science and Technology Program [grant nos. 2019 YFS0537 and 2020YFS0456]. 


\section{Authors' contributions}

Guarantors of the article: Jiahao Fan and Yong Xu.

JF and $Y X$ conceived, designed and supervised the study; $Y Z, X T, X F$ and TY provided research guidance; $\mathrm{XF}$ and HW collected the data and biological samples; XF and HW performed the measurements of serum maresin-1 levels; XF and HW analyzed the data and wrote the manuscript; and TY, XF, XT, YZ, JF and YX critically reviewed and edited the manuscript. All authors have read and approved the final manuscript.

\section{Acknowledgements}

The authors are grateful to all the participants for their participation.

\section{Authors' information}

${ }^{1}$ Department of Endocrinology and Metabolism, the Affiliated Hospital of Southwest Medical University, Luzhou, 646000, Sichuan, China. ${ }^{2}$ Cardiovascular and Metabolic Diseases Key Laboratory of Luzhou, 646000, Sichuan, China. ${ }^{3}$ Sichuan Clinical Research Center for Nephropathy, Luzhou, 646000, Sichuan, China. ${ }^{4}$ Department of Laboratory Medicine, the Affiliated Hospital of Southwest Medical University, Luzhou, 646000 , Sichuan, China. ${ }^{5}$ Department of Respiratory Medicine, Yongchuan Hospital of Traditional Chinese Medicine, Yongchuan, 402100, Chongqing, China. ${ }^{6}$ Department of Gastroenterology, the Affiliated Hospital of Southwest Medical University, Luzhou, 646000, China.

\section{References}

1. Wesolowski SR, Kasmi El, Jonscher KC, Friedman KR. JE. Developmental origins of NAFLD: a womb with a clue. Nat Rev Gastroenterol Hepatol. 2017;14(2):81-96. https://doi.org/10.1038/nrgastro.2016.160.

2. Younossi Z, Tacke F, Arrese M, Sharma BC, Mostafa I, Bugianesi E, et al. Global Perspectives on Nonalcoholic Fatty Liver Disease and Nonalcoholic Steatohepatitis. Hepatology. 2019;69(6):267282. https://doi.org/10.1002/hep.30251.

3. Feng RN, Du SS, Wang C, Li YC, Liu LY, Guo FC, et al. Lean-non-alcoholic fatty liver disease increases risk for metabolic disorders in a normal weight Chinese population. World J Gastroenterol. 2014;20(47):17932-40. https://doi.org/10. 3748/wjg.v20.i47.17932.

4. Zhu JZ, Zhou QY, Wang YM, Dai YN, Zhu J, Yu CH, et al. Prevalence of fatty liver disease and the economy in China: A systematic review. World J Gastroenterol. 2015;21(18):5695-706. https://doi.org/10.3748/wjg.v21.i18.5695.

5. Younossi Z, Anstee QM, Marietti M, Hardy T, Henry L, Eslam M, et al. Global burden of NAFLD and NASH: trends, predictions, risk factors and prevention. Nat Rev Gastroenterol Hepatol. 2018;15(1):11-20. https://doi.org/10.1038/nrgastro. 2017.109. 
6. Nishioji K, Sumida Y, Kamaguchi M, Mochizuki N, Kobayashi M, Nishimura T, et al. Prevalence of and risk factors for non-alcoholic fatty liver disease in a non-obese Japanese population, 2011-2012. J Gastroenterol. 2015;50(1):95-108. https://doi. org/10.1007/s00535-014-0948-9.

7. Younossi ZM, Blissett D, Blissett R, Henry L, Stepanova M, Younossi Y, et al. The economic and clinical burden of nonalcoholic fatty liver disease in the United States and Europe. Hepatology. 2016;64(5):1577-86. https://doi.org/10.1002/hep.28785.

8. Goldberg D, Ditah IC, Saeian K, Lalehzari M, Aronsohn A, Gorospe EC, et al. Changes in the Prevalence of Hepatitis C Virus Infection, Nonalcoholic Steatohepatitis, and Alcoholic Liver Disease Among Patients with Cirrhosis or Liver Failure on the Waitlist for Liver Transplantation. Gastroenterology. 2017;152(5):1090-9. https://doi.org/10.1053/j.gastro.2017.01.003.

9. Wong RJ, Aguilar M, Cheung R, Perumpail RB, Harrison SA, Younossi ZM, et al. Nonalcoholic steatohepatitis is the second leading etiology of liver disease among adults awaiting liver transplantation in the United States Nonalcoholic steatohepatitis is the second leading etiology of liver disease among adults awaiting liver transplantation in the United States. Gastroenterology. 2015;148(3):547-55. https://doi.org/10.1053/j.gastro.2014.11.039.

10. Mittal S, El-Serag HB, Sada YH, Kanwal F, Duan ZG, Temple S, et al. Hepatocellular Carcinoma in the Absence of Cirrhosis in United States Veterans is Associated with Nonalcoholic Fatty Liver Disease. Clin Gastroenterol Hepatol. 2016;14(1):124-31. https://doi.org/10.1016/j.cgh.2015.07.019.

11. Serhan CN, Dalli J, Karamnov S, Choi A, Park CK, Xu ZZ, et al. Macrophage proresolving mediator maresin 1 stimulates tissue regeneration and controls pain. FASEB J. 2012;6(4):1755-65. https://doi.org/10.1096/fj.11-201442.

12. Serhan CN, Yang R, Martinod K, Kasuga K, Pillai PS, Porter TF, et al. Maresins: novel macrophage mediators with potent antiinflammatory and proresolving actions. J Exp Med. 2009;206(1):15-23. https://doi.org/10.1084/jem.20081880.

13. Martínez-Fernández L, González-Muniesa P, Laiglesia LM, Sáinz N, Prieto-Hontoria PL, Escoté X, et al. Maresin 1 improves insulin sensitivity and attenuates adipose tissue inflammation in ob/ob and dietinduced obese mice. FASEB J. 2017;31(5):2135-45. https://doi.org/10.1096/fj.201600859R.

14. Laiglesia LM, Lorente-Cebrián S, Martínez-Fernández L, Sáinz N, Prieto-Hontoria PL, Burrell MA, et al. Maresin 1 mitigates liver steatosis in ob/ob and diet-induced obese mice. Int $\mathrm{J}$ Obes (Lond). 2018;42(3):572-9. https://doi.org/10.1038/ijo. 2017.226.

15. Jung TW, Kim HC, Abd El-Aty AM, Jeong JH. Maresin 1 attenuates NAFLD by suppression of endoplasmic reticulum stress via AMPK-SERCA2b pathway. J Biol Chem. 2018;293(11):3981-8. https://doi.org/10.1074/jbc.RA117.000885.

16. Han YH, Shin KO, Kim JY, Khadka DB, Kim HJ, Lee YM, et al. A maresin 1/RORa/12-lipoxygenase autoregulatory circuit prevents inflammation and progression of nonalcoholic steatohepatitis. J Clin Invest. 2019;129(4):1684-98. https://doi.org/10.1172/JCl124219.

17. Fan JG, Jia JD, Li YM, Wang BY, Lu LG, Shi JP, et al. Guidelines for the diagnosis and management of nonalcoholic fatty liver disease: update 2010: (published in Chinese on Chinese Journal of 
Hepatology 2010; 18:163-166). J Dig Dis. 2011;12 (1):38-44. https://doi.org/10.1111/j.17512980.2010.00476.x.

18. Wang $X$, Chen $X$, Zhang $H$, Pang J, Lin J, Xu X, et al. Circulating retinol-binding protein 4 is associated with the development and regression of non-alcoholic fatty liver disease. Diabetes Metab2020;46(2):119-28. https://doi.org/ 10.1016/j.diabet. 2019.04.009.

19. Alberti KGMM, Zimmet P, Shaw J. Metabolic syndrome-a new world-wide definition. A consensus statement from the International Diabetes Federation. Diabet Med. 2006;23(5):469-80. https://doi.org/10.1111/j.1464-5491.2006. 018 58.x.

20. Ni Y, Zhao LJ, Yu HY, Ma XJ, Bao YQ, Rajani C, et al. Circulating Unsaturated Fatty Acids Delineate the Metabolic Status of Obese Individuals. EBioMedicine. 2015;2(10):1513-22. https://doi.org/10.1016/j.ebiom.2015.09.004.

21. Miao T, Huang BL, He NX, Sun LH, Du GS, Gong XL, et al. Decreased Plasma Maresin 1 Concentration Is Associated with Diabetic Foot Ulcer. Mediators Inflamm. 2020;2020:4539035. https://doi.org/10.1155/2020/4539035.

22. Rombaldova M, Janovska P, Kopecky J, Kuda O. Omega-3 fatty acids promote fatty acid utilization and production of pro-resolving lipid mediators in alternatively activated adipose tissue macrophages. Biochem Biophys Res Commun. 2017;490(3):1080-5. https://doi.org/10.1016/j.bbrc.2017.06.170.

23. Tang S, Wan M, Huang W, Stanton RC, Xu Y. Maresins. Specialized Proresolving Lipid Mediators and Their Potential Role in Inflammatory-Related Diseases. Mediators Inflamm. 2018;2018:2380319. https://doi.org/10.1155/2018/2380319.

24. Sasaki K, Urabe D, Arai H, Arita M, Inoue M. Total synthesis and bioactivities of two proposed structures of maresin. Chem Asian J. 2011;6(2):534-43. https://doi. org/10.1002/asia.201000494.

25. Tosello-Trampont AG, Landes SG, Nguyen V, Novobrantseva TI, Hahn YS. Kuppfer cells trigger nonalcoholic steatohepatitis development in diet-induced mouse model through tumor necrosis factor-a production. J Biol Chem. 2012;287(48):40161-72.

https://doi.org/10.1074/jbc.M112.417014.

26. Wehr A, Baeck C, Ulmer F, Gassler N, Hittatiya K, Luedde T, et al. Pharmacological inhibition of the chemokine CXCL16 diminishes liver macrophage infiltration and steatohepatitis in chronic hepatic injury. PLoS One. 2014;9(11):e112327. https://doi.org/10.1371/journal.pone.0112327.

27. Samuel VT, Shulman GI. Nonalcoholic Fatty Liver Disease as a Nexus of Metabolic and Hepatic Diseases. Cell Metab. 2018;27(1):22-41. https://doi.org/10. 1016/j.cmet.2017.08.002.

28. Kim NH, Kim JH, Kim YJ, Yoo HJ, Kim HY, Seo JA, et al. Clinical and metabolic factors associated with development and regression of nonalcoholic fatty liver disease in nonobese subjects. Liver Int. 2014;34(4):604-11. https://doi.org/10. 1111/ liv.12454.

29. Hao Y, Zheng H, Wang RH, Li H, Yang LL, Bhandari S, et al. Maresin1 Alleviates Metabolic Dysfunction in Septic Mice: A ${ }^{1}$ H NMR-Based Metabolomics Analysis. Mediators Inflamm. 2019;2019:2309175. https://doi.org/10.1155/2019/2309175. 
30. Martínez-Fernández L, González-Muniesa P, Sáinz N, Escoté X, Martínez JA, Arbones-Mainar JM, et al. Maresin 1 regulates insulin signaling in human adipocytes as well as in adipose tissue and muscle of lean and obese mice. J Physiol Biochem. 2021;77(1):167-73.

https://doi.org/10.1007/s13105-020-00775-9.

31. Félix-Soriano E, Sáinz N, Gil-Iturbe E, Collantes M, Fernández-Galilea M, Castilla-Madrigal R, et al. Changes in brown adipose tissue lipid mediator signatures with aging, obesity, and DHA supplementation in female mice. FASEB J. 2021;35(6):e21592.

https://doi.org/10.1096/fj.202002531R.

32. Markworth JF, Brown LA, Lim E, Castor-Macias JA, Larouche J, Macpherson PCD, et al. Metabolipidomic profiling reveals an age-related deficiency of skeletal muscle pro-resolving mediators that contributes to maladaptive tissue remodeling. Aging Cell. 2021;20(6):e13393. https://doi.org/10.1111/acel.13393.

33. Friedman SL, Neuschwander-Tetri BA, Rinella M, Sanyal AJ. Mechanisms of NAFLD development and therapeutic strategies. Nat Med. 2018;24(7):908-22. https://doi.org/10.1038/s41591-018-01049.

\section{Figures}

A

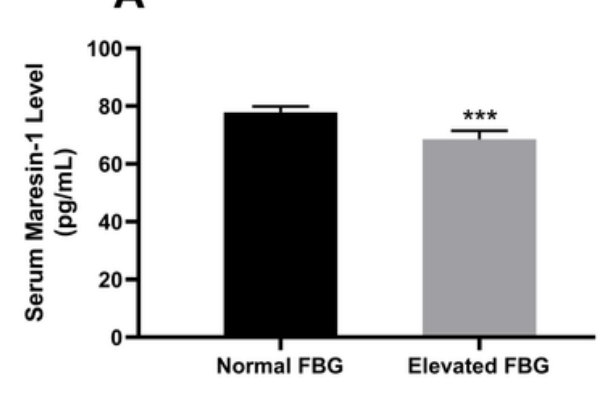

D

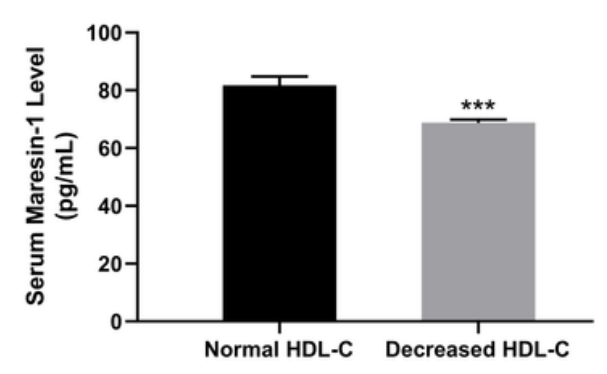

B

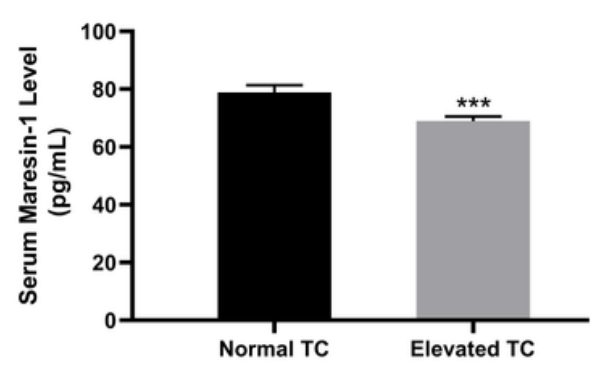

E

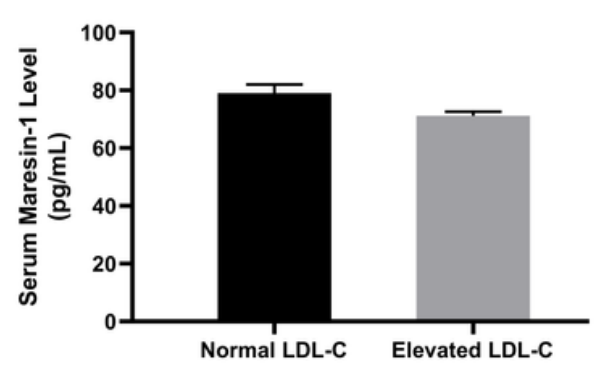

C

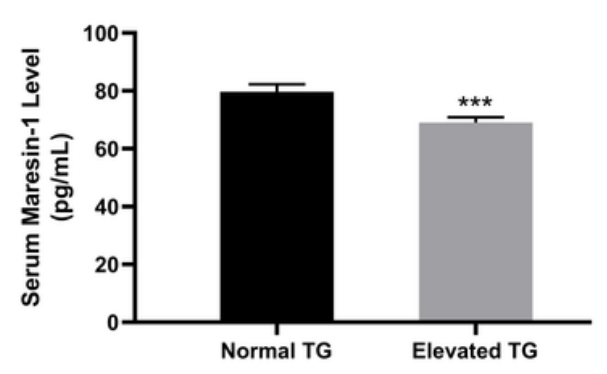

$\mathbf{F}$

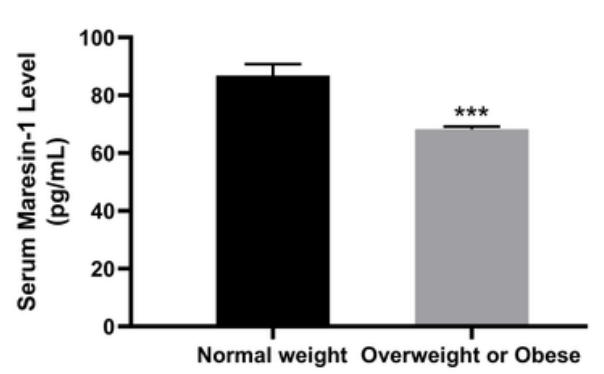

Figure 1 
Serum Maresin-1 concentration for study population $(A)$ with $(n=63)$ or without elevated FBG levels $(n=$ $177)$, (B) with ( $n=84)$ or without elevated TC levels $(n=156),(C)$ with $(n=97)$ or without elevated TG levels $(n=143)$, (D) with $(n=117)$ or without decreased HDL-C levels $(n=123)$, $(E)$ with $(n=112)$ or without elevated LDL-C levels $(n=128),(F)$ with normal weight $(n=92)$ or with overweight or obesity $(n=$ 148). ${ }^{*} P<0.05$, ${ }^{\star * P}<0.01,{ }^{* \star} P<0.001$ versus without elevated FBG, TC, TG or LDL-C levels or without decreased HDL-C levels or with normal weight. Data are expressed as means \pm SEM.

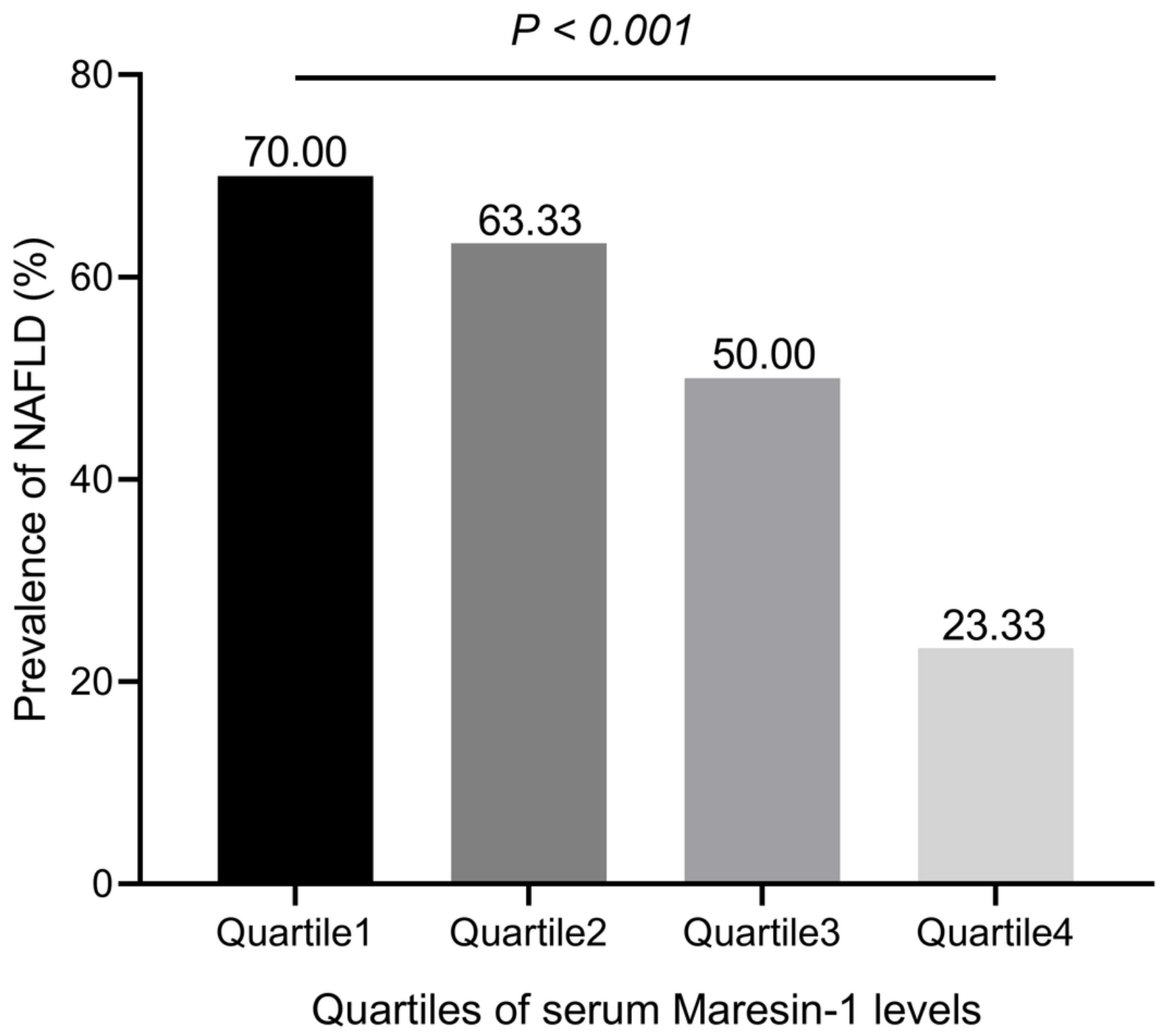

Figure 2

Prevalence of non-alcoholic fatty liver disease (NAFLD) by quartiles of serum Maresin-1 in all participants. 